\title{
Transtorno do Espectro Autista: intervenção psicopedagógica a partir de "self"
}

\section{Natália Hends de Medeiros ${ }^{1}$, Luciane Corte Real ${ }^{2}$, Antonina Garcia Cavalheiro ${ }^{3}$, Ana Amália Roveda ${ }^{4}$, Gabriela Ferraz ${ }^{5}$, Denise Favarim ${ }^{6}$, Lívia Ferreira ${ }^{7}$}

${ }^{1}$ Tutora a distância - Universidade Federal do Rio Grande do Sul - Porto Alegre - RS, Brasil.

${ }^{2}$ Professora - Faculdade de Educação, Universidade Federal do Rio Grande do Sul - Porto Alegre - RS, Brasil.

${ }^{3}$ Professora - Prefeitura Municipal de Restinga Seca - Restinga Seca - RS, Brasil.

${ }^{4}$ Educadora Especial - Escola Estadual de Educação Básica General Osório- Ibirubá - RS, Brasil.

${ }^{5}$ Professora - Escola Municipal de Ensino Fundamental Vila Monte Cristo - Porto Alegre RS, Brasil.

${ }^{6}$ Professora - Escola Técnica Estadual Parobé - Porto Alegre - RS, Brasil.

${ }^{7}$ Educadora Especial - Fundação Catarinense de Educação Especial - Florianópolis - SC, Brasil.

\footnotetext{
natalia hends@yahoo.com.br, lucreal@gmail.com, antonina.uabrestinga@gmail.com, nanaroveda@hotmail.com, gabifr@gmail.com, parobe.denisefavarim@gmail.com, livia.edesp@gmail.com
}

Resumo O artigo trata de um estudo de caso, exploratório, que envolve Psicopedagogia e Tecnologias Digitais. O objetivo foi investigar um aluno da Educação Infantil com o diagnóstico de Transtorno do Espectro Autista a partir do uso das tecnologias digitais. A investigação faz parte de uma pesquisa na Universidade Federal do Rio Grande do Sul que estuda o Autismo e as Tecnologias Digitais. Foram realizadas observações, entrevistas e intervenções. Os autores que orientaram a coleta e análise de dados foram as pesquisas de Jean Piaget e Alícia Fernandez. Os resultados apontaram que o uso do "self" no tablet foi um meio de comunicação entre o aluno, sua família e seus colegas.

Palavras-chave: Autismo, Tecnologias Digitais, Psicopedagogia.

\begin{abstract}
The article is a case study, exploratory, involving and Psychopedagogy and Digital Technologies. The objective was to investigate a student from kindergarten diagnosed with Autism Spectrum Disorder from the use of digital technologies. The research is part of a research in a Universidade Federal do Rio Grande do Sul studying autism and Digital Technologies. Observations, interviews and interventions were performed. Authors who guided the collection and analysis of data were Jean Piaget and Alicia Fernandez. The results showed that the use of "self " in the tablet was a means of communication between the student, his family and his colleagues .
\end{abstract}

Keywords: Autism, Digital Technologies, Psychopedagogy. 
V Congresso Brasileiro de Informática na Educação (CBIE 2016)

Anais dos Workshops do V Congresso Brasileiro de Informática na Educação (CBIE 2016)

\section{Apresentação}

Na busca da compreensão da forma como as Tecnologias de Informação e Comunicação (TIC) contribuem para o fazer psicopedagógico a ser realizado com alunos com Transtorno do Espectro Autista (TEA) tem-se como escopo investigar se através das TIC, do uso de computadores e da internet com seus diferentes recursos metodológicos, é possível melhorar o desenvolvimento da pessoa que apresenta TEA e partilhar os diferentes olhares psicopedagógicos no apoio a elas.

Para tanto, inicialmente, descreve-se sobre o autismo, sua trajetória e especificidades. A seguir aborda-se o trabalho psicopedagógico com as TIC, contextualizando-as com a linha de pesquisa trabalhada no curso de especialização em Psicopedagogia e Tecnologias da Informação e Comunicação da Universidade Federal do Rio Grande do Sul. Para então, partilhar relatos evidenciados sobre o trabalho psicopedagógico realizado com portadores de TEA, as estratégias utilizadas através das TIC e as considerações em relação ao desenvolvimento da aprendizagem.

\section{Autismo, Tecnologias Digitais e trabalhos afins}

O Transtorno do Espectro Autista ou simplesmente chamado de autismo foi descrito pela primeira vez por Kanner em 1943. Já nesta época existia um consenso em torno do entendimento sobre o que caracterizava o autismo, ou seja, eram os aspectos observáveis que indicavam problemas na comunicação e na interação social, além de comportamentos repetitivos e de áreas restritas de interesse (AMA, 2011). É considerado um transtorno no desenvolvimento que impossibilita a pessoa de se relacionar com as outras pessoas, muitas vezes, com um isolamento completo.

A pessoa diagnosticada de Transtorno do Espectro Autista apresenta prejuízo nas áreas de interação, comunicação, imaginação, podendo variar em graus de comprometimento. Wehmuth e Antoniuk (2013, p.26) afirmam que "autismo não é uma doença única, mas sim um distúrbio de desenvolvimento complexo, definido de um ponto de vista comportamental, com causas múltiplas e graus variados de comportamento". Conhecer os aspectos inerentes ao TEA torna-se fundamental para propor qualquer intervenção.

Alguns estudos têm demonstrado que a utilização de TIC com crianças diagnosticadas autistas vêm contribuindo para o seu desenvolvimento. Passerino (2005) acompanhou dois grupos de sujeitos com autismo em atividades de interação, mediadas em diferentes ambientes digitais e concluiu que estratégias de mediações adequadas e adaptadas a cada sujeito foram importantes para o desenvolvimento cognitivo e social dos sujeitos com autismo.

Em sua tese de doutorado, Bez (2014) desenvolveu um sistema de comunicação alternativa (Sistema SCALA) para web e dispositivos móveis gratuitos, com o objetivo de apoiar a inclusão de sujeitos com autismo, de 3 a 5 anos, em diferentes contextos sociais. Já Foscarini (2013) fez uso do Sistema SCALA com três sujeitos com autismo, não oralizados e evidenciou que a utilização desse sistema contribuiu para o surgimento de novos gestos e a significação dos olhares, apontamentos, sorrisos e outras demonstrações interativas.

A pesquisa desenvolvida por Pires (2014) teve como objetivo avaliar se as TIC, nas suas mais variadas formas, poderiam ser vantajosas no ensino/educação de autistas. Os resultados deste estudo revelaram que o sucesso educativo do aluno com TEA pode ser melhor 
caso se recorra frequentemente as TIC_ainda que elas tenham que ser devidamente adaptadas, acompanhadas e adequadas às necessidades específicas do aluno.

Sousa et al (2012) apresentam o uso de software dentro de suas interfaces adaptativas no apoio ao desenvolvimento cognitivo de crianças autistas com atividades lúdicas envolvendo sequências de planejamento. Passerino et al (2006) sustenta como um estudo multicasos com observação de grupos de autistas nas atividades de interação mediadas por Ambientes Digitais de Aprendizagem ao longo de 3 anos e apontaram categorias relevantes emergentes dessa mediação tecnológica com descobertas significativas nas áreas da Psicologia, Educação e Computação. Já Bittencourt e Francisco (2015) utilizam o blog no trabalho com o TEA como uma constituição de um espaço de expressão, de apropriação das tecnologias da informação e comunicação e aprendizagem dos recursos informatizados, sendo as atividades desenvolvidas uma aprendizagem compartilhada, uma partilha de experiências, socialização, interação, melhorias no raciocínio e comunicação, resgate da autoestima, sentimento de utilidade e satisfação. Junior e Junior (2015) fizeram uma proposta de 'teatro virtual' apresentando profissionais mediadores (terapeutas) em um ciclo de avaliação formativa identificando a adequação do propósito e o potencial para apoiar a ação junto a crianças com TEA.

As Tecnologias Digitais podem contribuir para a comunicação e relacionamento dos portadores de TEA. Estudos vêm demonstrando como a comunicação alternativa pode ter uma ação potencializadora no desenvolvimento de sujeitos com autismo, tornando-os cada vez mais incluídos tanto no ambiente escolar, na sociedade e na vida de uma forma geral. Cardoso (2015, p. 105) afirma que:

Na visão psicopedagógica, especialistas defendem que esta inclusão é importante para que as pessoas não sejam socializadas para a incapacidade, a restrição, a dependência e ao desrespeito. Todas as pessoas têm o direito de viver e conviver com seres sociáveis, e a escola é uma ponte para fazer essa ligação com a sociedade, para incluir estas pessoas.

Se, através da educação pudessem ser trabalhadas questões relacionadas à linguagem, à responsabilidade social e à capacidade adaptativa dessas crianças [Gardin, 2006], oportunizariam o prosseguimento aos conceitos tradicionais trabalhados no ambiente escolar. Para tanto, a comunicação alternativa torna-se fundamental, uma vez que pode possibilitar a comunicação e a interação de sujeitos com TEA com o mundo ao seu redor.

\section{Psicopedagogia e Aprendizagem}

Parte-se da premissa que a aprendizagem precisa ser algo desejado pelo sujeito, ou seja, precisa ser um processo prazeroso, de curiosidade e de inquietação constante. Conforme uma entrevista da pesquisadora Alícia Fernandez (2011):

a atividade do pensar é fascinante e "a fábrica" de pensamentos não se situa nem dentro, nem fora da pessoa, está localizada entre [...] A atividade do pensar nasce na intersubjetividade promovida pelo desejo de se fazer 
próprio que nos é desconhecido, mas também nutrida pela necessidade de entendermos e de que nos entendam.

Segundo Alícia Fernandez (1990, p. 70) "este profissional estuda o sujeito epistêmico que se refere à coordenação geral das ações (reunir, ordenar, etc.) constitutivas da lógica, e não o sujeito individual que se refere a ações próprias e diferenciadas de cada individuo". Ou seja, cada sujeito vai se desenvolvendo conforme sua estrutura lógica e a dimensão de desejo e compreende-se que a criança vai desenvolvendo sua inteligência, por exemplo, no domínio de conservação de matéria, uma criança precisa de certo amadurecimento para conseguir compreender tal conceito, se não o faz com quatro anos não está errada, apenas está em processo de amadurecimento cognitivo.

Acredita-se então, que o psicopedagogo precisa munir-se de estratégias de aprendizagem voltadas a essas duas questões: estrutura lógica e funções desejantes de cada aluno.

Segundo Fernandez (1990, p. 126) "é, para nós, fundamental encontrar o original, o particular, o apaixonante de cada história" É possível afirmar que o "escutar e o olhar do terapeuta vai permitir ao paciente falar e ser reconhecido, e ao terapeuta compreender a mensagem" [Fernandez, 1999, p. 131].

\section{Metodologia}

Trata-se de uma pesquisa qualitativa e exploratória na forma de estudo de caso. Conforme Yin (2001) o estudo de caso é uma estratégia de pesquisa que compreende vários procedimentos para a coleta e análise de dados. Na presente investigação foi realizada observações, entrevistas e intervenções. O estudo foi exploratório e trata-se de um subprojeto de uma pesquisa da Faculdade de Educação da Universidade Federal do Rio Grande do Sul que estuda o "Autismo e Tecnologias". As teorias que orientaram a coleta e análise dos dados foi a epistemologia genética de Jean Piaget e Alícia Fernandez.

Segundo Fernandez (1991) o olhar e a escuta psicopedagógica durante as intervenções ocorrem paralelamente ao atendimento, de forma que, para propor atividades que promovam benefícios à criança, é necessário, entre outras coisas, "incorporar uma atitude clínica e nestas estão incluídos conhecimentos sobre como se aprende e sobre o organismo [..] saber sobre o aprender e o não aprender" (p. 128). Ou seja, à medida que se compreende o perfil de aprendizagem da criança, o canal pelo qual apresenta melhores respostas, se entende as limitações ou condições inerentes ao transtorno e pode-se conduzir de maneira mais efetiva as intervenções mediando o processo de desenvolvimento.

No livro "Seis estudos de psicologia", Piaget (1999, p.13 traz contribuições importantes sobre o desenvolvimento psíquico que são base para compreesão da psicopedagogia:

O desenvolvimento psíquico, que começa quando nascemos e termina na idade adulta, é comparável ao crescimento orgânico: como este, orienta-se, essencialmente, para o equilíbrio. Da mesma maneira que um corpo está em evolução até atingir um nível relativamente estável - caracterizado pela conclusão do crescimento e pela maturidade dos órgãos -, também a vida 
mental pode ser concebida como evoluindo na direção de uma forma de equilíbrio final, representada pelo espírito adulto.

É sujeito desta pesquisa um aluno da Educação Infantil com idade de 5 anos com o diagnóstico de TEA de uma escola municipal do interior do Rio Grande do Sul. Foram realizados sete encontros entre a pesquisadora e o aluno com o objetivo de se aproximar do caso estudado e levantar qual ou quais as tecnologias que poderiam ser utilizadas com a criança.

Os responsáveis assinaram o Termo de Consentimento Livre e Esclarecido e a criança o Termo de Assentimento.

\section{Apresentação dos dados e resultados}

Penetrar no mundo reservado e muitas vezes indiferente de um autista exige estudos, reflexões, técnicas e metodologias sendo a maior dificuldade a interação e a comunicação como um dos primeiros de muitos desafios a serem enfrentados.

Nos encontros com a criança, pode-se evidenciar um novo comportamento motivado pelo prazer de utilizar tecnologias para novas descobertas. $\mathrm{O}$ aluno demonstrou interesse pelo celular e pelo tablet com os quais tirava fotos, olhava vídeos e explorava os aparelhos de acordo com suas possibilidades. A partir desse interesse que a pesquisadora utilizou-se das fotos "self" tiradas pela criança no tablet como fonte para acompanhar o seu desenvolvimento realizando intervenções psicopedagógicas. O aluno mostrava as fotos com emoção, abandonando por alguns minutos sua indiferença e seu isolamento. $\mathrm{O}$ interesse em tirar fotos selfs e mostrar a psicopedagoga revelou um convite da criança para a pesquisadora para participar do seu processo de aprendizagem. Através do interesse da criança pela foto self percebeu-se uma grande oportunidade de contribuir desenvolvimento desta. Construiu-se através das fotos selfs recursos para o olhar e a escuta psicopedagógica durante as intervenções

Assim com as fotos selfs foram feitas gravações que também foram apreciadas pela criança. Foi filmada sua apresentação na escola e o aluno filmou a dos demais colegas. A criança assiste concentrada as apresentações muitas vezes ao dia.

Durante os encontros, a pesquisadora incentivou a criança a partilhar suas descobertas por meio dos registros que produzia no tablet. A criança ao longo dos encontros demostrou interesse em querer mostrar os vídeos a outras pessoas. Em outros momentos apontava no tablete sua imagem demostrando contentamento com as interações construídas com a psicopedagoga.

No relato de sua mãe, quando ia para o sítio de seu vovô pedia para fotografar e filmar suas atividades e brincadeiras com os animais para assim partilhar com a pesquisadora. Demostrava interesse em manusear o tablet para rever as filmagens dos registros das suas vivencias com a pesquisadora assim como o interesse em partilhar as aprendizagens construídas.

Observou-se que ao longo dos encontros a criança ficou mais carinhosa, mesmo que, muitas vezes, passe do carinho à agressão, com a maior naturalidade. As fotos "self" registradas no tablet assim outras gravações permitiram uma melhora no desenvolvimento da criança quanto a sua autoimagem e relação com os colegas de aula. 
Na pesquisa realizada identificou-se que a escola, os meios de comunicação, a música, o celular e o tablet abriram um novo mundo para criança. Foi por meio das fotos "selfs", de suas filmagens e das de seus familiares, que foi possibilitada nova forma de comunicação, tornando a criança mais participativa na escola, dentro de suas limitações e de seu quadro de autismo. Dessa maneira evidencia-se a importância do apoio psicopedagógico com o TEA e o uso de Tecnologias Digitais.

\section{Considerações finais ou iniciais}

Considerando o caso investigado como estudo exploratório, as considerações finais do artigo são consideradas iniciais para futuras investigações.

O desenvolvimento típico de aprendizagem acontece através das interações desde o contato visual do bebê com a mãe até as brincadeiras com pais, familiares, professores e colegas. E a habilidade na aquisição de novas aprendizagens e a capacidade de estabelecer relações ocorre através da interação, imitação e comunicação desenvolvendo assim aspectos cognitivos e motores. No desenvolvimento da criança com TEA os déficits nas áreas que ajudam a promover essas habilidades fazem com que o processo de aprendizagem ocorra de forma diferenciada.

Segundo Cunha,

Há uma relação diferente entre o cérebro e os sentidos, e as informações nem sempre se tornam em conhecimentos. Os objetos não exercem atração em razão da sua função, mas em razão do estimulo que promovem [...] A criança precisa aprender a função de cada objeto e seu manuseio adequado (2010, p. 31).

Nessa perspectiva, muitos comportamentos e habilidades passam a ser considerados importantes e a ter maior valor pedagógico sendo incluídos nos planejamentos das intervenções. O principal objetivo da intervenção foi melhorar as possibilidades de comunicação, interação social, interesses, autonomia e uso da imaginação do sujeito (através de brincar com o "self"). Tais habilidades possibilitaram aprendizagem e possibilidade de inclusão no grupo.

Considerando as Tecnologias Digitais no apoio psicopedagógico observou-se avanços após as intervenções com estes recursos. O resultados alcançados podem ser considerados não apenas pelo uso da tecnologia, mas também pelo vínculo afetivo que foi necessário ser construído ao longo das intervenções.

A partir deste estudo, foi possível refletir na importância do conhecimento psicopedagógico e do uso de material tecnológico nas intervenções do profissional especializado. Reforça-se, no caso do autismo, as ações e ferramentas necessárias para a evolução da linguagem, da sociabilidade e da integração da pessoa com seus iguais.

Os estudos que envolvem o TEA têm crescido nos últimos anos, porém, dentro da área da Psicopedagogia com uso das tecnologias ainda se apresentam insuficientes, nesse sentido, estudos que busquem investigar o TEA em uma abordagem psicopedagógica tecnológica podem ser oportunidades de inovações em pesquisa. 
V Congresso Brasileiro de Informática na Educação (CBIE 2016)

Anais dos Workshops do V Congresso Brasileiro de Informática na Educação (CBIE 2016)

\section{Referências}

AMA - Associação de Amigos do Autista, 2011. Disponível em: <http://www.ama.org.br/site/definicao.html>. Acesso em: 14 de janeiro de 2016.

ANTONIUK, Sérgio Antonio. (Org.). Autismo Perspectivas no Dia a Dia. Curitiba: Ithala, 2013.

BEZ, Maria Rosangela. SCALA : Sistema de comunicação alternativa para processos de inclusão em autismo: uma proposta integrada de desenvolvimento em contextos para aplicações móveis e web. Porto Alegre: UFRGS, 2014. 286 f. Tese de Doutorado - Programa de Pós Graduação em Informática na Educação. Universidade Federal do Rio Grande do Sul, Porto Alegre, 2014.

BITTENCOURT, Ivanise. FRANCISCO, Deise. Os efeitos da produção em blog para sujeitos com Transtorno do Espectro Autista. Anais do SBIE 2015. Disponível em: <http://www.brie.org/pub/index.php/sbie/article/view/5368/3731>. Acesso em: maio. 2016.

CARDOSO, Marilene. A escola inclusiva e a prática na sala de recursos multifuncional: implicações psicopedagógicas.In: CAIERÃO, Iara; KORTMANN, Gilca Lucena (org.). A prática psicopedagógica: processos e percursos do aprender. Rio de Janeiro: Wak editora, 2015. p. $99-112$.

CUNHA, Eugênio. Autismo e Inclusão. $2^{\circ}$ Ed. - Rio de Janeiro: Wak Ed., 2010.

FERNÁNDEZ, Alícia. A inteligência aprisionada. Porto alegre: Artes Médicas, 1990.

FERNANDEZ, A. A inteligência aprisionada. Porto Alegre: Artmed, 1991.

FERNANDEZ, Alicia. Entrevista: Alicia Fernández, Agosto, 2011. Disponível em:

<http://www.abpp.com.br/publicacoes_entrevista_alicia_fernandez.html>. Acesso em: abril. 2016.

FOSCARINI, Ana Carla. A intencionalidade de comunicação mediada em autismo : um estudo de aquisição de gestos no sistema SCALA. Porto Alegre: UFRGS, 2013. 156 f. Dissertação de Mestrado - Programa de Pós Graduação em Educação. Universidade Federal do Rio Grande do Sul, Porto Alegre, 2013.

GADIA, Carlos. Aprendizagem e autismo. In: Transtornos da aprendizagem / Newra Tellechea Rotta... [et al.]. - Porto Alegre: Artmed, 2006.

JUNIOR, Osvaldo Tavares Viana. JUNIOR, Alberto Nogueira de Castro. Um Esquema para Autoria de Histórias em Mundos Virtuais. Anais do SBIE 2016.

Disponível em: <http://www.br-ie.org/pub/index.php/sbie/article/view/5386/3747>. Acesso em: maio. 2016. 
V Congresso Brasileiro de Informática na Educação (CBIE 2016)

Anais dos Workshops do V Congresso Brasileiro de Informática na Educação (CBIE 2016)

PASSERINO, Liliana Maria. Pessoas com autismo em ambientes digitais de aprendizagem : estudo dos processos de interação social e mediação. Porto Alegre: UFRGS, 2005. 316 f. Tese de Doutorado - Programa de Pós Graduação em Informática na Educação. Universidade Federal do Rio Grande do Sul, Porto Alegre, 2005.

PASSERINO, Liliana M. (Org.) Pessoas com Autismo em Ambientes Digitais de Aprendizagem: estudo dos processos de Interação Social e Mediação. Anais do SBIE 2006. Disponível em: <http://www.br-ie.org/pub/index.php/sbie/article/view/465/451 >. Acesso em: maio. 2016.

PIRES, Raquel Pinho. O Impacto das TIC no Sucesso Educativo de Alunos com Autismo. Lisboa: Escola Superior de Educação João de Deus, 2014. 107 f. Dissertação de Mestrado Programa de Pós Graduação em Ciências da Educação na Especialidade de Educação Especial: Domínio Cognitivo e Motor. Escola Superior de Educação João de Deus, Lisboa, 2014.

PIAGET, Jean. Seis estudos de psicologia.24 ed. Rio de Janeiro: Forense Universitária, 1999.

SOUSA Felipe Rodrigues Monteiro (Org.) WorldTour: Software para Suporte no Ensino de Crianças Autistas. Anais do SBIE 2012. Disponível em:

<http://www.brie.org/pub/index.php/sbie/article/view/1806/1567> . Acesso em: maio. 2016.

YIN, Robert K. Estudo de caso: planejamento e métodos / Robert K. Yin; trad. Daniel Grassi - 2.ed. -Porto Alegre: Bookman, 2001. 